\title{
FIBROUS DYSPLASIA OF PROXIMAL PHALANX OF HAND A RADIOLOGICAL DIAGNOSIS AND HISTOPATHOLOGICAL CONFIRMATION
}

\author{
Shobha Khanduri ${ }^{1}$, Sachin Khanduri², Umesh C. Parashari ${ }^{3}$, Saakshi Chhabra ${ }^{4}$, Samarjit Bhadury ${ }^{5}$
}

\section{HOW TO CITE THIS ARTICLE:}

Shobha Khanduri, Sachin Khanduri, Umesh C. Parashari, Saakshi Chhabra, Samarjit Bhadury. "Fibrous Dysplasia of Proximal Phalanx of Hand A Radiological Diagnosis and Histopathological Confirmation". Journal of Evolution of Medical and Dental Sciences 2014; Vol. 3, Issue 21, May 26; Page: 5882-5885,

DOI: $10.14260 /$ jemds/2014/2677

\begin{abstract}
Fibrous dysplasia is a benign skeletal developmental anomaly, which is manifested as a defect in osteoblastic differentiation and maturation of a normal bone that leads to structurally weak fibrous and osseous (bone-like) tissue. In normal bone formation, woven bone appears first and later matures into lamellar bone and in fibrous dysplasia, bone does not mature and development stops in the woven bone stage. Most commonly involved bones are femur, tibia, pelvis, ribs and skull. Small bones of hands or feet are rarely involved. This disorder is usually diagnosed in childhood or early adulthood and can affect one or several bones. Histopathology reveals abundant amount of fibrous tissue and disorganized bony trabeculae and spindle cells. Normal bone pattern is not demonstrated. Here we report a very rare case of fibrous dysplasia involving proximal phalanx of the hand diagnosed on radiological features with histopathological confirmation.
\end{abstract}

KEYWORDS: Fibrous dysplasia, monostotic, polyostotic, x-ray, histopathology.

INTRODUCTION: Monostotic fibrous dysplasia of the hand is rare. Fibrous dysplasia is an abnormal bone growth in which the normal bone is replaced by fibrous tissue. This disorder is usually diagnosed in childhood or early adulthood and can affect one or several bones. Males and females of any race are equally affected. There are two forms of fibrous dysplasia. Monostotic form involves one bone and presents at the age of 10-70 years. The bones commonly involved are ribs, femur, tibia, mandible, skull and humerus.

Phalanx is an uncommon site and very few cases have been reported in literature. Lesions do not increase over time and disease becomes inactive at puberty. Polyostotic form involves many of few bones and involves larger segment of the bone. It is frequently associated with fractures and severe deformity. Most common complications are pain associated with pathological fracture. Malignant transformation is rare but if occurs it can lead to osteosarcoma, fibrosarcoma and chondrosarcoma.

Radiological features are pathognomic. Histopathology reveals abundant amount of fibrous tissue and disorganized bony trabeculae and spindle cells surrounded by fibrous matrix. Surgery is indicated for confirmatory biopsy and correction of deformity.

CASE REPORT: A 14 years old female came to the hospital with a painful swelling of the index finger in the left hand. The swelling had been present since she was 5 years old and increasing in size for the past 2 years. There was no history of trauma to the finger or overlying discoloration of the skin.

There was full range of movement between the interphalangeal joint. X-ray findings showed an intramedullary expansile well-defined area of radiolucency with hazy ground glass appearance with maintained smooth cortical contour. Endosteal scalloping of overlying cortex was also noted. No 
evidence of any soft tissue swelling was noted. (Figure 1) Despite being an uncommon site for fibrous dysplasia the diagnosis was made and later the diagnosis was confirmed on histopathological study.

Curettage of the lesion was performed in addition to the resection of the dorsal cortex, which was thin and fragile. On histopathology, (H \& E, magnification 10x) showed benign fibrous stroma with woven bony trabeculae showing no specific pattern and no osteblastic rimming (Figure 2). Another slide ( $\mathrm{H}$ \& E, magnification 40x) showed spindle cell proliferation with interspersed irregular osteoid with no osteoblastic rimming (Figure 3). Thus confirming the diagnosis of monostotic fibrous dysplasia of the phalanx of the left hand.

DISCUSSION: Fibrous dysplasia is among the most common benign skeletal disorder in which the normal bone marrow is replaced by fibro-osseous tissue. ${ }^{1}$ FD most often affects adolescents and young adults. FD can be monostotic or polyostotic; monostotic form is seen in $70 \%$ of cases. ${ }^{2}$ The skull and facial bones are involved in $25 \%$ of patients with monostotic FD and $40 \%$ to $60 \%$ of patients with the polyostotic form. Polyostotic FD is most commonly found in the femur, tibia, pelvis, and foot. Monostotic form never converts to polyostotic form.

FD involving proximal femur is prone to repeated fractures that can result in a bowing deformity with varus angulation called as "shepherd's deformity". Skull involvement may cause neurological deficits such as visual and hearing loss. Leontiasisossea and Cherubism are the forms of cranio facial deformity that involves the facial and mandible respectively. ${ }^{3}$ This case is rare because phalanx is an uncommon site and the radiological picture was pathognomic and they were confirmed on histopathology.

Clinical symptoms may vary from being asymptomatic in monostotic FD to bone pain, swelling, tenderness, facial deformity to cranial nerve palsies in symptomatic patients. FD expands and replaces the normal bony medullary spaces with vascular fibrocellular tissue. Malignant transformation is present in only $0.4-4 \%$ of the cases. Most common tumors are osteosarcoma, fibrosarcoma and chondrosarcoma. ${ }^{4}$

The rate of malignant transformation is higher for polyostotic lesions than for monostotic lesions. Diagnosis of FD can be made on the basis of radiological picture. On plain radiographs and CT fibrous dysplasia appears as a well-defined intramedullary expansile radiolucent lesion with ground glassing to completely sclerotic lesion in the diaphysis and metaphysis. Endosteal scalloping of the overlying cortex is commonly seen. MR imaging is done to see the extent and content of the lesion. The signal varies from intermediate to low intensity on T1 weighted images and intermediate to high on T2 weighted images and heterogeneous contrast enhancement suggestive of mainly fibrous and bone components. ${ }^{5}$

When the diagnosis is suspected on CT or MRI, histological confirmation by bone biopsy or surgical resection is necessary. Histologically, disorganized bony trabeculae and spindle cells surrounded by fibrous matrix with overgrowth of fibrous tissue are noted. The fibrous tissue is usually of low cellularity and contains variable amounts of myxoid material to dense collagenous matrix. Hemorrhage and cystic change may be occasionally found. ${ }^{6}$

There are various syndromes that are associated with fibrous dysplasia. McCune-Albright Syndrome (MAS) is a rare multisystem disorder characterized by a triad of polyostotic fibrous dysplasia, precocious puberty and abnormal cutaneous pigmentation i.e., café au lait spots. ${ }^{7}$ Mazabraud syndrome is a rare syndrome comprising of polyostotic fibrous dysplasia and multiple 
soft tissue intramuscular myxoma. It is more common in females and there is increased risk of osseous malignant transformation. ${ }^{8}$ The differential diagnosis may include ossifying-fibroma, giant cell tumor, osteoma, bone cyst or a malignancy of osseous origin. ${ }^{9}$ The recurrence rate of fibrous dysplasia after curettage and bone grafting is high. The monostotic form does not convert to the polyostotic form.

For treatment of FD especially in polyostotic form where surgery is not possible bisphosphonate therapy is indicated for the reduction of pain. ${ }^{10}$ Surgical procedures are required for correction of deformities, prevention of pathologic fractures, or eradication of symptomatic lesions.

The choice of the surgical procedures has been controversial. Some performed curettage with or without cancellous bone graft and others do en bloc resection with cortical bone graft.

CONCLUSION: Monostotic fibrous dysplasia of the hand is rare and the involvement of the phalanx is even rarer. Clinicians should have a high index of suspicion for diagnosing this rare entity via relevant investigations. Radiological features give us a clear picture about the lesion and histopathology plays a very important role in confirming the diagnosis. Though there is still a dilemma regarding surgical procedure of choice, yet it is required for palliation.

\section{REFERENCES:}

1. Eisenberg RL. Bubbly lesions of bone. AJR Am J Roentgenol. Aug 2009; 193 (2):W79-94.

2. Godse AS, Shrotriya SP, Vaid NS. Fibrous dysplasia of the maxilla. J Pediatr Surg. Apr 2009; 44 (4):849-51.

3. Ozek C, Gundogan H, Bilkay U, et al. Craniomaxillofacial fibrous dysplasia. J Craniofac Surg2002; 13: $382-389$.

4. Shah ZK, Peh WC, Koh WL, et al. Magnetic resonance imaging appearances of fibrous dysplasia. Br J Radiol. Dec 2005; 78 (936):1104-15.

5. Wootton-Gorges SL. MR imaging of primary bone tumors and tumor-like conditions in children. Magn Reson Imaging Clin N Am. Aug 2009; 17 (3):469-87.

6. Kahn MA, Lucca M, Papageorge MB. A clinico-pathologic correlation: fibrous dysplasia. J Mass Dent Soc. Winter 2006; 54 (4):48-50.

7. Campanacci M. Bone and soft tissue tumors: clinical features, imaging, pathology and treatment, 2nd ed. Wien, Austria: Springer, 1999:435 -460.

8. Iwasko N, Steinbach LS, Disler D, et al. Imaging findings in Mazabraud's syndrome: seven new cases. Skeletal Radiol 2002; 31: 81 -87.

9. Parekh SG, Donthineni-Rao R, Ricchetti E, et al. Fibrous dysplasia. J Am Acad Orthop Surg 2004; 12: 305-13.

10. Saglik Y, Atalar H, Yildiz Y, et al. Management of fibrous dysplasia. A report on 36 cases. Acta Orthop Belg. Feb 2007; 73 (1):96-101. 


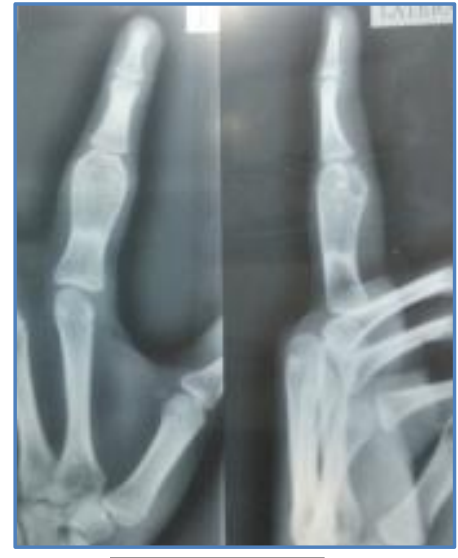

Figure 1

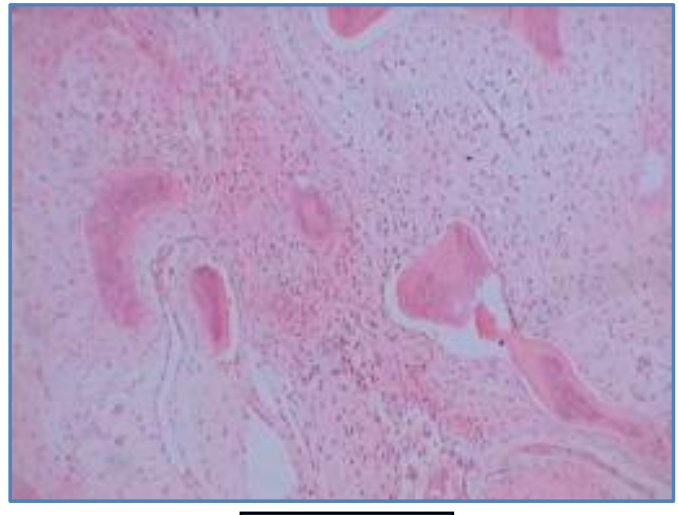

Figure 2

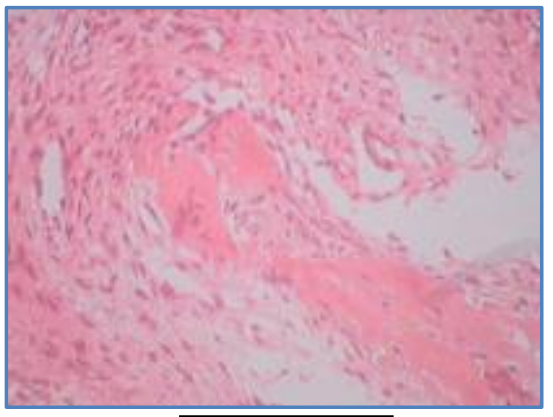

Figure 3

\section{AUTHORS:}

1. Shobha Khanduri

2. Sachin Khanduri

3. Umesh C. Parashari

4. Saakshi Chhabra

5. Samarjit Bhadury

\section{PARTICULARS OF CONTRIBUTORS:}

1. Lab Head \& Pathologist, Department of Pathology, S.R.L. Ltd. Lucknow.

2. Professor, Department of Radiodiagnosis, Era's Medical College and Hospital, Lucknow.

3. Associate Professor, Department of Radiodiagnosis, Era's Medical College and Hospital, Lucknow.

4. Resident, Department of Radiodiagnosis, Era's Medical College and Hospital, Lucknow.
5. Professor, Department of Radiodiagnosis, Era's Medical College and Hospital, Lucknow.

\section{NAME ADDRESS EMAIL ID OF THE CORRESPONDING AUTHOR:}

Dr. Sachin Khanduri, \#C-149,

Indiranagar,

Lucknow.

Email: sasho0622@hotmail.com

Date of Submission: 25/04/2014.

Date of Peer Review: 26/05/2014.

Date of Acceptance: 13/05/2014.

Date of Publishing: 26/05/2014. 